\title{
Correction to: \\ ABM Clinical Protocol \#29: Iron, Zinc, and Vitamin D Supplementation During Breastfeeding, by Sarah N. Taylor and The Academy of Breastfeeding Medicine. Breastfeed Med 2018;13(6):398-404. DOI: 10.1089/bfm.2018.29025.snt
}

\footnotetext{
T $\mathrm{N}$ The July/August 2018 issue of Breastfeeding Medicine (vol. 13, no. 6; 398-404), the article titled ABM Clinical Protocol \#29: Iron, Zinc, and Vitamin D Supplementation During Breastfeeding by Sarah N. Taylor and The Academy of Breastfeeding Medicine requires a correction because the author inadvertently added the word "not" to the second sentence in the first paragraph on page 398:
}

"However, micronutrient supplementation may not be appropriate, especially when a mother is deficient or an infant has special needs such as prematurity."

The sentence should have appeared as:

However, micronutrient supplementation may be appropriate, especially when a mother is deficient or an infant has special needs such as prematurity.

The online version of the article has been corrected to reflect this change.

Sarah N. Taylor apologizes for the error. 